\title{
PLGA NANOPARTICLES LOADED MUCOADHESIVE AND THERMOSENSITIVE HYDROGEL AS A POTENTIAL PLATFORM FOR THE TREATMENT OF ORAL MUCOSITIS
}

\author{
GINA S. EL-FEKY*a, GAMAL M. ZAYED ${ }^{\mathrm{b}, \mathrm{c}}$
}

aDepartment of Pharmaceutical Technology, National Research Center, bDepartment of Pharmaceutics and Industrial Pharmacy, Faculty of Pharmacy, Al-Azhar University at Assiut, Egypt, cAl-Azhar Centre of Nanosciences and Applications (ACNA), Assiut, Egypt Email: gelfeky@hotmail.com

Received: 01 Sep 2018, Revised and Accepted: 19 Nov 2018

\section{ABSTRACT}

Objective: The objective of this study was to design an effective topical treatment for oral mucositis.

Methods: Poly-(DL-lactide-co-glycolide) (PLGA) nanoparticles (NPs) and Poloxamer407 (PLX)/Hydroxy propyl methyl cellulose (HPMC) hydrogel matrix (HG) were used as combined carriers for benzydamine HCL (BNZ). BNZ loaded PLGA nanoparticles were assessed for their particle size, PDI, zeta potential and entrapment efficiency. Scanning electron microscopy, thermosensitivity study, mucoadhesion study, in vitro release and in vivo investigation were used to characterize the combined BZN loaded PLGA NPs HG.

Results: Negatively charged NPs with an average diameter of $139 \pm 4.92 \mathrm{~nm}$ were incorporated into PLX/HPMC HG bases. The gelation temperature of BZN-PLGA-NPs-HGs ranged between $31^{\circ} \mathrm{C}$ and $36.5^{\circ} \mathrm{C}$. When diluted with saliva simulated fluid, BZN-PLGA-NPs-HGs preserved their gelation properties. Mucoadhesion was found lower for formulations prepared with PLX without HPMC. An increase in the concentrations of PLX from 10 to $30 \%$ resulted in an increase in adhesion. Both PLGA-NPs and PLGA-NPs-HG provided a biphasic drug release profile while BZN-HG provided monophasic zero order release pattern. The in vivo study showed that animal groups treated with BZN-HG and BZN-PLGA-NPs-HG showed a significantly higher reduction percentage in ulcer surface area compared to those treated with BZN-PLGA-NPs. BZN-PLGA-NPs-HG group needed 10 $\mathrm{d}$ of treatment to complete healing versus $16 \mathrm{~d}, 14 \mathrm{~d}$ and $12 \mathrm{~d}$ for the complete healing of groups with no treatment, treated with BZN-PLGA-NPs and treated with BZN-HG, respectively.

Conclusion: BZN-PLGA-NPs-HG could represent a promising mean for the effective treatment of oral mucositis induced by cancer therapy.

Keywords: Mucositis, PLGA nanoparticles, Thermosensitive hydrogel, Mucoadhesive hydrogel, In vivo

(C) 2019 The Authors. Published by Innovare Academic Sciences Pvt Ltd. This is an open access article under the CC BY license (http://creativecommons.org/licenses/by/4.0/) DOI: http://dx.doi.org/10.22159/ijap.2019v11i1.29466

\section{INTRODUCTION}

Mucositis is a highly common side effect to cancer treatments [1]. It is characterized by painful lesions and odynophagia. Mucositis often degrades patients' quality of life and affects compliance with anticancer therapies to the extent of interrupting treatment [2-4]. Oral mucositis is reported in about $100 \%$ of patients on high-dose chemotherapy, in $80 \%$ of patients with head and neck malignancies and who are treated with radiotherapy and in $40 \%$ of patients receiving conventional doses of cytostatic chemotherapy [5]. To our knowledge, there is no approved method for either the prevention or the treatment of mucositis [2] except for some therapeutic strategies which depends on the use of direct cytoprotectants such as laser, sucralfate, corticosteroids, silver nitrate, vitamins and antioxidants, cryotherapy and indirect cytoprotectants including antimicrobial agents and haematopoietic growth factors [2]. BNZ $\mathrm{HCl}$ is a nonsteroidal drug that possesses analgesic, anesthetic, antiinflammatory, antipyretic and antimicrobial properties [6]. BZN is suggested to exert its action through suppressing selected proinflammatory cytokine production which is hypothesized to be one major mechanism of oral mucositis [6]. $\mathrm{BZN} \mathrm{HCl}$ is used as a mouthwash or mouth spray in a concentration of $0.15 \%$ [7], however, such forms show a short residence time in the buccal cavity, therefore, the concentration of BZN $\mathrm{HCl}$ on the oral mucosa cannot be maintained, and in turn, it is difficult to expect an effective treatment.

Over the past few decades, there has been considerable interest in the development of biodegradable polymeric drug carriers. PLGA is one of the most attractive biodegradable synthetic polymers approved by the FDA for human use [8-10], for its safety profile [8], biocompatibility, as well as its tunable mechanical properties and ability to effectively deliver the drug to the target site following a controllable degradation into metabolite monomers, glycolic acid and lactic acid [8] which are further metabolized by the body via the Krebs cycle $[11,12]$. However, when PLGA NPs are applied directly to the buccal mucosa, they are drained rapidly through the salivary action, which leads to their rapid elimination through involuntary swallowing $[13,14]$. For this reason, the development of mucoadhesive preparations for buccal administration of PLGA nanoparticles is important. HG with mucoadhesive and thermosensitive properties represent a promising option, since mucoadhesion allow close contact with the buccal mucosa, providing adhesiveness and prolonging the residence time of the drug $[15,16]$ and in situ thermosensitivity allows easy application and quick spreading [17] of the formulation onto the site of action at room temperature whereas, the subsequent gelation enhance the hydrogel's mucoadhesive property. Pluronic $₫ /$ poloxamers are a group of polymers which exhibits thermos-reversible property in aqueous solutions. Poloxamer 407 is widely used as a gelling agent in the concentration range of 20 to $30 \%$. Poloxamer 407 not only can self-assemble to form micelles but is also known to form gels in situ in response to a temperature increase [18-21]. However, they generally have a high critical gelation concentration (CGCs) and poor resilience [22], in addition, the hydrogels of poloxamer 407 formed above the sol gel transition temperature generally exhibit no or poor bioadhesive properties [23]. Therefore, formulating poloxamer 407 with other bioadhesive polymers such hydroxyl-propylmethylcellulose (HPMC), will decrease the used concentration, increase its mucoadhesion and in turn enhance its ability to withstand salivation, tongue movements and swallowing for a significant period of time [24].

The aim of the study was to evaluate the impact of BZN-PLGA-NP-HG on the treatment of oral mucositis in vivo.

\section{MATERIALS AND METHODS}

\section{Materials}

Benzydamine Hydrochloride, a kind gift from EIPICO, Egypt. Hydroxy propyl methyl cellulose, a kind gift from EIPICO, Egypt. Poloxamer 407, Purchased from Sigma, USA. Poly (lactic-co-glycolic) 
polymer; 50:50 (PLGA 50:50) was a kind gift from PURAC $^{\circledR}$. Other materials were of analytical grade.

\section{Preparation of free (PLGA NPs) and BZN HCl loaded PLGA nanoparticles (BZN-PLGA-NPs)}

The porous PLGA nanoparticles loaded with BNZ HCl were prepared using the water-oil-water emulsion solvent evaporation method. Briefly, $100 \mathrm{mg}$ PLGA were dissolved in $4 \mathrm{ml}$ dichloromethane and placed in an ice bath, then $0.8 \mathrm{ml}$ of distilled water was added to the PLGA solution dropwise. The resulting mixture was emulsified by ultra-sonication in an ice bath for $2 \mathrm{~min}$, after which a solution of ammonium bicarbonate $(100 \mathrm{mg} / \mathrm{ml}, 0.1 \mathrm{ml})$ was added and the mixture was re-sonicated in an ice bath for $30 \mathrm{~s}$ to form the primary emulsion. The primary emulsion was poured into $50 \mathrm{ml}$ of poly-(vinyl alcohol) aqueous solution $(0.5 \%, \mathrm{w} / \mathrm{v})$ and homogenized at $3000 \mathrm{rpm}$ in an ice bath for $3 \mathrm{~min}$. The resulting emulsion was added to $50 \mathrm{ml}$ of distilled water, and the dichloromethane was allowed to evaporate under magnetic stirring at room temperature over $5 \mathrm{~h}$. Finally, the formed nanoparticles were collected by centrifugation at $5000 \mathrm{rpm}$ for $15 \mathrm{~min}$, washed with distilled water three times, and then lyophilized. The dried porous nanoparticles were stored at -20 until use.

For the preparation of BNZ-PLGA-NPs, a specific amount of the drug equivalent to $0.15 \% \mathrm{w} / \mathrm{v}$ final concentration was added to distilled water before addition to the PLGA dichloromethane solution and further steps were continued as mentioned above.

\section{Characterization of PLGA nanoparticles}

\section{Particle size, PDI and zeta potential}

The particle size, PDI and zeta potential of drug-free PLGA NPs and BNZ-PLGA-NPs were determined by dynamic light scattering (DLS) using a Zetasizer (Malvern Instrument, Worcestershire, UK). All samples were diluted with distilled water and equilibrated for 30 min before measurement.

\section{Entrapment efficiency (EE)}

A specific weight of freeze-dried BNZ-PLGA-NPs was soaked in $10 \mathrm{ml}$ phosphate buffer for 30 min using bath sonicator. The solution was then centrifuged at $15000 \mathrm{rpm}$ in at $15{ }^{\circ} \mathrm{C}$ for $30 \mathrm{~min}$ to remove polymeric debris. The supernatant was collected, and the polymeric debris were washed twice with fresh buffer to extract any adhered drug. The clear supernatant solution was then analyzed for its BZN content using UV spectrophotometer at $\lambda$ max of $305 \mathrm{~nm}$.

Complete extraction of the drug was confirmed by repeating the extraction process on the already extracted polymeric debris. Entrapment efficiency was calculated by using the following equation:

$\% \mathrm{EE}=\frac{\text { (Amount of drug initially used to prepare the PLGANPs)-(Amount of drug released from the PLGANPS) }}{\text { (Amount of drug initially used to prepare the PLGANPs) }} * 100$

\section{Preparation of HG bases}

Six different in situ gel formulations were prepared (table 1). PLX was used either alone (in three different concentrations) or in combination with HPMC in formulating HGs. Different weight ratios of PLX: HPMC were investigated to assess the effect of adding HPMC on the thermosensitive and mucoadhesive properties of PLX. For the preparation of PLX HGs (F0), 10 g Poloxamer 407 were dissolved in 50 $\mathrm{ml}$ distilled water under magnetic stirring and left in the refrigerator for $24 \mathrm{~h}$. For the preparation of PLX/HPMC HG, HPMC was added in each specific concentration to the formed PLX HG, continuous stirring was applied till all components were completely dissolved and the formed hydrogel was left in the refrigerator for further $24 \mathrm{~h}$.

\section{Preparation of BNZ-PLGA-NPs loaded hydrogel}

The BNZ-PLGA-NPs dispersion previously optimized was incorporated into the blank HG bases prepared using high speed stirring for $3 \mathrm{~min}$ at $1000 \mathrm{rpm}$, in a concentration of $50 \%(\mathrm{w} / \mathrm{w})$ of the BNZ-PLGA-NPs dispersion into HG. The final concentration of BNZ in all BNZ-PLGA-NPs-HG formulation was set to $0.15 \% \mathrm{w} / \mathrm{v}$.

Table 1: Composition of the prepared free HG formulations

\begin{tabular}{|c|c|c|c|}
\hline Formulation & PLX (g) & Drug Concentration (\%) & HPMC (g) \\
\hline F0 & 10 & 0.15 & 0 \\
\hline F00 & 20 & 0.15 & 0 \\
\hline F000 & 30 & 0.15 & 0 \\
\hline F1 & 10 & 0.15 & 0.25 \\
\hline $\mathrm{F} 2$ & 10 & 0.15 & 0.5 \\
\hline F3 & 10 & 0.15 & 1 \\
\hline
\end{tabular}

\section{Evaluation of hydrogel bases}

\section{Scanning electron microscopy (SEM)}

The morphology of F3 BZN-PLGA-NPs-HG was examined using scanning electron microscopy (JSM-5900LV, JEOL, Japan). Before observation, the sample was freeze-dried and was cut-off with a treatment of liquid nitrogen.

\section{Thermosensitivity study}

The "gelation temperature" method was used to assess the temperature responsive gelation property of the prepared hydrogels. A sample solution was put in a $250 \mathrm{ml}$ beaker, the beaker was then placed in a water bath heated slowly from $20^{\circ} \mathrm{C}$ to $40{ }^{\circ} \mathrm{C}$ at an increment of $2{ }^{\circ} \mathrm{C} / \mathrm{step}$ under magnetic stirring. The gelation point was determined using a stopwatch when the magnetic bar stopped rotating, the temperature at which the magnetic bar stopped moving was determined as the gelation temperature.

To evaluate the effect of dilution by buccal saliva on the thermosensitive gelation capacity of the in situ BZN-PLGA-NPs-HGs, similar measurements as mentioned above were carried out but after mixing the hydrogel with simulated saliva fluid (SSF) at the volume ratio of 5:1. Simulated saliva was prepared according to the following composition $8 \mathrm{~g} / \mathrm{l}$ sodium chloride, $0.19 \mathrm{~g} / \mathrm{lpotassium}$ phosphate monobasic and $2.38 \mathrm{~g} / \mathrm{l}$ sodium phosphate dibasic $[25,26]$.

\section{Mucoadhesion evaluation}

Mucin-interaction method was used to evaluate the mucoadhesion properties of the prepared hydrogels through determining the changes in zeta potential. Porcine mucin powder was hydrated in demineralized water at $4{ }^{\circ} \mathrm{C}$ for $12 \mathrm{~h}$. The mucin solution was then adjusted to $\mathrm{pH} 7.4$ and diluted to a final concentration of $1 \%(\mathrm{w} / \mathrm{v})$ using $0.1 \mathrm{M}$ phosphate buffer ( $\mathrm{pH}$ 7.4). The mucin solution was centrifugated at $5000 \mathrm{rpm}$ for $30 \mathrm{~min}$, the resulting supernatant was filtered and used in the mucoadhesion experiment. Equal volumes of each prepared hydrogel, one at a time, and the $1 \%(\mathrm{w} / \mathrm{v})$ mucin particles solution were mixed using a vortex for 1 min. The zeta potential of each of the mucin solution and the mixtures was measured using Zetasizer and the changes in the zeta potential following interaction of the hydrogel with the negatively charged mucin [27] was investigated.

\section{In vitro drug release}

The in vitro release characteristics of $\mathrm{BNZ} \mathrm{HCl}$ from $\mathrm{HG}$ matrix, PLGA-NPs and PLGA-NPs-HG were studied. Samples of each of the tested formulations containing predetermined drug content of $0.15 \% \mathrm{w} / \mathrm{w}$ were placed first in a dialysis bag then in a dissolution apparatus containing $350 \mathrm{ml}$ phosphate buffer of $\mathrm{pH} 6.8 \pm 0.5$ at $37 \pm 0.5{ }^{\circ} \mathrm{C}$. The stirring speed was set to $50 \mathrm{rpm}$. Aliquots of $2 \mathrm{ml}$ were withdrawn at previously specified time intervals and replaced 
by equal volumes of fresh buffer solution. Samples were measured spectrophotometrically at $\lambda \max 305 \mathrm{~nm}$ [28].

\section{In vivo animal study}

The in vivo protocol was carried out in accordance with the Guidelines laid down by the National Institute of Health (NIH) in the USA regarding the care and use of animals for experimental procedures and in accordance with local laws and regulations. Twenty-four male albino rabbits weighing 2.5-3.0 kg provided by the animal house of Assiut University, Egypt. Animal ethical approval was obtained from the Ethics Committee of the Faculty of Pharmacy, Al-Azhar University (approval code pharmaceut05) were used for this study. Animals have been acclimatized for one week. Each group of rabbits was placed in a separate cage with free access to food and drink. The cage environment was controlled to optimum humidity and $25^{\circ} \mathrm{C}$ temperature.

Oral mucositis was induced by cancer chemotherapy [29]. Rabbits were administered an intraperitoneal injection of 5-fluorouracil on 5,3 and $1 \mathrm{~d}$ prior to the experiment at a dose of $50 \mathrm{mg} / \mathrm{kg}$ body weight. On the day of the experiment, mice were anesthetized, and the model of oral mucositis was enhanced through creating aseptic tissue necrosis in all 24 animals using round filter paper soaked in $15 \mathrm{ml}$ of $50 \%$ acetic acid. In order to create round ulcers and facilitate measurements and follow up, the acid-soaked paper was pressed onto the labial gingival tissue of the rabbits for $60 \mathrm{~s}$. Animals were divided into four groups, each of 6 animals; Group 1: Control group where animals received no treatment, Group 2: where animals received BZN-HG treatment, Group 3: where animals received BNZ-PLGA-NPs treatment and Group 4: where animals received BNZ-PLGA-NPs-HG treatment. The treatment started $24 \mathrm{~h}$ after ulcer initiation (day 1). The dose of BZN $\mathrm{HCl}$ used for groups 2 and 3 was $1 \mathrm{mg} / \mathrm{kg}$ drug applied twice daily. The area of the ulcer measured on day 1 before treatment application was considered the baseline reading. The progress and healing of the buccal ulcer were evaluated by subtracting the daily reading from the ulcer measurement at day 1 .

\section{Statistical analysis}

The statistical analysis was carried out using Graphpad Software Instat (version 6; GraphPad Software, Inc., La Jolla, CA, USA). The level of significance was set at probability $* \mathrm{P}<0.05$.

\section{RESULTS AND DISCUSSION}

\section{PLGA nanoparticles}

Free and BZN loaded PLGA nanoparticles were prepared and characterized for their physicochemical properties including particle size, PDI and zeta potential. BZN-PLGA-NPs were further characterized for their entrapment efficiency (\%) (table 2). Experimental conditions for the preparation of PLGA nanoparticles including the sonication time and amplitude were optimized after preliminary trials (data not shown) to $100 \%$ amplitude and $12 \mathrm{~min}$ sonication time [30].

The particle size of BZN free PLGA-NPs was found to be $69.27 \pm 5.19$ $\mathrm{nm}$ while that of BZN-PLGA-NPs was found to be a bit higher $(90.81$ $\pm 4.92 \mathrm{~nm}$ ), similar particle size ranges for free and loaded PLGA NPs were previously reported [31, 32]. PDI showed uniform size distribution. Zeta potential of both free and BZN loaded PLGA-NPs were found to be-29.17 and-26.51, respectively indicating the formation of stable particles. Both formulations showed a negative charge with BZN-PLGA-NPs showing slightly lower values than free PLGA NPs. Negative values of zeta potential obtained for PLGA-NPs might be due to the presence of terminal carboxyl groups in the polymer. The percentage of incorporated BZN in the polymeric matrix was found to be $80 \%$ which agrees with the findings of Abrego et al., conducted in 2014 [33].

Table 2: Particle size, PDI, zeta potential and entrapment efficiency of PLGA-NPs

\begin{tabular}{lll}
\hline & Free PLGA & BZN-PLGA-NPs \\
\hline Particle Size (nm) & $69.27 \pm 5.19^{*}$ & $90.81 \pm 4.92^{*}$ \\
PDI & 0.197 & 0.265 \\
Zeta Potential (mv) & -29.17 & -26.51 \\
EE (\%) & - & $79.92 \pm 3.10^{*}$ \\
\hline
\end{tabular}

${ }^{*}$ Results are expresses as mean $\pm \mathrm{SD}, \mathrm{n}=3$.

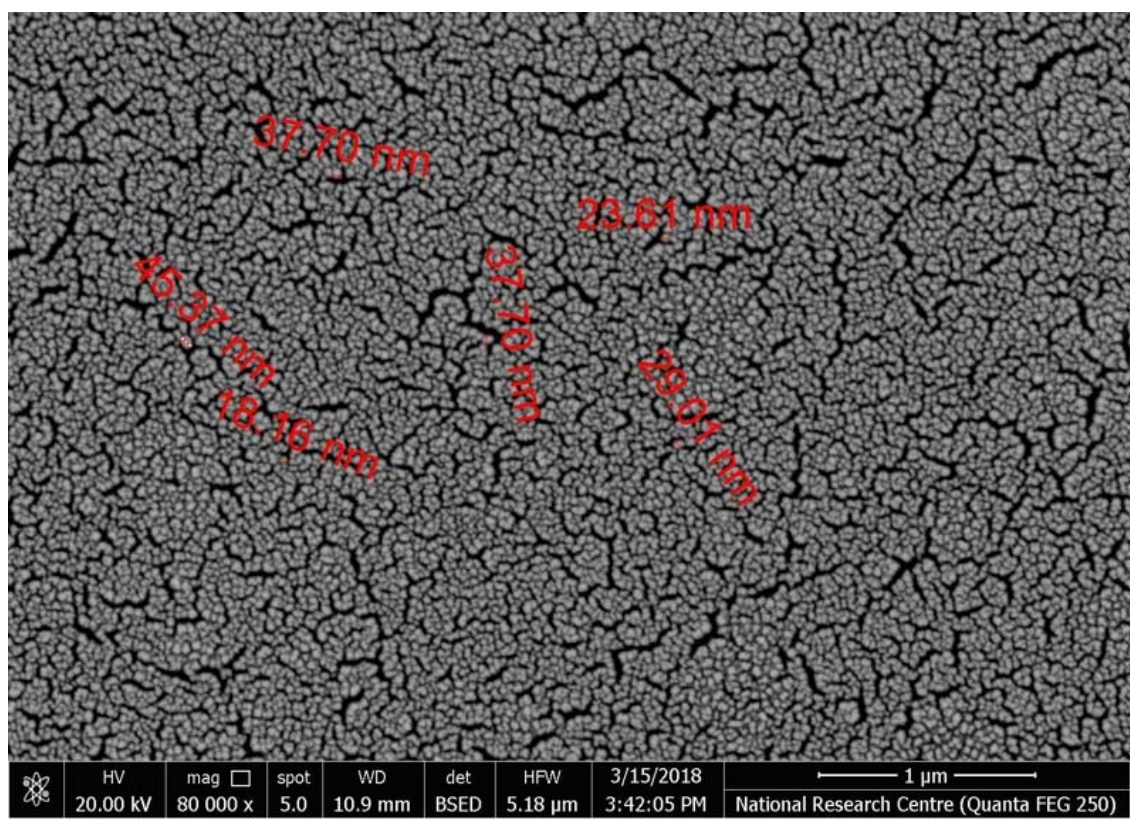

Fig. 1: SEM micrograph of BZN-PLGA-NPs-HGs 


\section{Preparation and characterization of PLX/HPMC HG}

HPMC together with PLX 407 were used in different w/w ratios to prepare a set of three different formulations of buccal in situ gels (table 1). BZN-PLGA-NPs were incorporated into different HG bases and the properties of BZN-PLGA-NPs-HGs were assessed.

\section{Scanning electron microscopy (SEM)}

Fig. 1 reveals the distribution of PLGA-NPs within the porous channels of HG matrix. SEM image shows the nanosized dimensions of PLGA-NPs.

\section{Thermosensitivity study}

According to the results shown in table 2, the gelation temperature of all BZN-PLGA-NPs-HGs ranged between $31^{\circ} \mathrm{C}$ and $36.5^{\circ} \mathrm{C}$ which renders them all suitable for buccal application [34]. However, it was obvious that the general range of gelation temperatures was higher for HGs formed of PLX solely which reflects the effect of mixing HPMC with PLX on reducing the gelation temperature.

To study the effect of HPMC addition on minimizing the concentration of PLX used, different concentrations of HPMC were mixed with the lowest tested concentration of PLX (10 gm). Interestingly, gelation temperature of PLX/HPM Cmixtures (F1, F2 and F3) were modulated to lower values compared to PLX solutions alone (F0, F00 and F00) which suggests that HPMC had a synergistic effect on the gelation of PLX. It has been previously reported that the incorporation of HPMC increased the elastic character of poloxamer gels and decreased their temperature of gelation [23]. PLX is a triblock copolymer which when present in aqueous solution and with increasing temperature, the hydrophobic chains of the copolymer undergo de-solvation due to the breaking of hydrogen bonds previously formed between the solvent and these chains. This promotes hydrophobic interactions among the polyoxypropylene domains and leads to gel formation [35] and at the definite point of gelation, micelles come into contact and organize under a micellar cubic phase [36] and no longer move. Micelle entanglements do not allow micelles to separate easily from each other and thus, forms rigid HG [37]. In the presence of HPMC, the HPMC chains might be able to bridge the poloxamer micelles, thus, leading to the formation of a network of interconnected micelles. This bridging might occur as early as micellization starts and even before the organization of the micelles under a cubic phase [23]. HPMC; through its highly hygroscopic nature, and its multiple hydroxyl functional groups, enhances the dehydration of PLX and, consequently the hydrophobic interactions between the poly-(propylene oxide) blocks.

The BZN-PLGA-NPs-HG formulations were mixed with SSF to simulate expected dilution in the in vivo circumstances. As shown in table 3, when diluted with $1 \mathrm{ml} \mathrm{SSF}$, all BZN-PLGA-NPs-HGs were found to preserve their gelation properties with non-significant differences in gelation temperature reported with any BZN-PLGANPs-HG before and after addition of SSF $\left({ }^{*} p>0.05\right)$. All BZN-PLGANPs-HGs retained gelation temperatures below $37{ }^{\circ} \mathrm{C}$ except for formula F0 containing 10\% PLX/0\% HPMC which showed a gelation temperature of $37.3^{\circ} \mathrm{C}$ (table 3 ).

Table 3: Gelation time measurements of different formulations

\begin{tabular}{llllll}
\hline Formulation & PLX (gm) & Drug concentration (\%) & HPMC (gm) & Gelation ${ }^{*}$ temp $\left({ }^{\circ} \mathbf{C}\right)$ & Gelation \\
\hline F0 & temp after addition of SSF $\left({ }^{\circ} \mathbf{C}\right)$ \\
F00 & 10 & 0.15 & 0 & $36.5 \pm 0.45$ & $37.3 \pm 1.16$ \\
F000 & 20 & 0.15 & 0 & $35 \pm 0.84$ & $35.6 \pm 0.28$ \\
F1 & 30 & 0.15 & 0 & $34 \pm 0.79$ & $34.5 \pm 1.29$ \\
F2 & 10 & 0.15 & 0.25 & $32.5 \pm 0.73$ & $33.3 \pm 0.65$ \\
F3 & 10 & 0.15 & 0.5 & $32.5 \pm 1.01$ & $33.2 \pm 0.82$ \\
\hline
\end{tabular}

${ }^{*}$ Results are expressed as mean $\pm \mathrm{SD}, \mathrm{n}=3$.

\section{Mucoadhesion study}

As stated by Chatterjee and his colleagues in 2017, the success of mucoadhesive formulations depends on the ability of the polymer/s to retain at the mucous layer and to sustain the drug release [44]. Mucoadhesive formulations contain at least one hydrophilic polymer, when this polymer comes in contact to saliva, due to the aqueous nature of saliva, it is wetted, and simultaneously adheres to the mucous with some physical interaction [38]. The polymers employed in BNZ-PLGA-NPs-HG formulations have been denoted as mucoadhesive and, therefore, it would be anticipated that the formulation would display good adherence properties [39-40].

The mucin-particle method was used to evaluate the mucoadhesive properties of BZN-PLGA-NPs-HG formulations through measuring the changes in the zeta potential of mucin following the addition of different BZN-PLGA-NPs-HGs one at a time at $\mathrm{pH}$ 6.8. The in vitro mucoadhesion test confirmed that all formulations possessed mucoadhesive properties where a significant change $\left({ }^{*} \mathrm{P}<0.5\right)$ in the zeta potential of mucin with BNZ-PLGA-NPs-HG compared to free mucin was reported for all formulations (table 4).
Mucoadhesion was found lower for formulations prepared with PLX without HPMC i.e., F0, F00 and F000 with F0 containing 10 gm PLX/0 gm HPMC showing the lowest mucoadhesive property, however, an increase in the concentrations of PLX from 10 to $30 \%$ resulted in an increase in adhesion. On the other hand, the presence of HPMC with its known mucoadhesive characteristics [41] enhanced mucoadhesion with F3; containing the highest concentration of HPMC; showing the highest mucoadhesive property. It's worth mentioning that the addition of HPMC to PLX enhanced the mucoadhesion property of the HG inspite of the lower concentration of PLX used which highlights the synergistic influence of the concomitant presence of PLX and HPMC in the HG. The mucoadhesive force increased in formulations F1, F2 and F3 with increasing HPMC concentration from $0.25 \%$ to $1 \%$ [42]. Cellulose derivatives such as HPMC are considered first generation mucoadhesive polymers [43], they acquire their adhesion property through the formation of $\mathrm{H}$ bond between the carboxylic acid group of the cellulose polymer and the glycoprotein of mucin. As the concentration of HPMC in the formulation increases, stronger $\mathrm{H}$ bonds are formed causing deeper and stronger attachment of the HG with the mucous layer [44]. Mucoadhesion results are likely to ensure a prolonged adhesion of the HGs at the mucosal surface, following their buccal administration.

Table 4: Effect of the interaction of hydrogel and mucin on Zeta potential

\begin{tabular}{ll}
\hline Material tested & Zeta potential (mV) \\
\hline Mucin & -20.98 \\
Mucin+F0 & 14.84 \\
Mucin+F00 & 12.19 \\
Mucin+F000 & 11.51 \\
Mucin+F1 & 10.01 \\
Mucin+F2 & 7.43 \\
Mucin+F3 & 5.29 \\
\hline
\end{tabular}




\section{In vitro drug release}

The release of drug from three different formulations was performed; HG, PLGA-NPs and PLGA-NPs-HG. Based on the thermo sensitivity and mucoadhesion results, the HG composed of $10 \mathrm{~g}$ PLX and $1 \mathrm{~g}$ HPMC was selected as the base of HG and PLGA-NPs-HG formulations used in the in vitro release experiment.

As shown in fig. 2, both PLGA-NPs and PLGA-NPs-HG formulations offered a slower release of BZN when compared to HG. PLGA-NPsHG provided the slowest release of BZN amongst the three tested formulations. The fact that PLGA-NPs were retained within the PLX/HPMC HG matrix might have influenced the release profile of BZN and reduced the initial burst release of the drug molecules [45].

Both PLGA-NPs and PLGA-NPs-HG provided a similar biphasic drug release profile [46]. Compared to BZN-PLGA-NPs, BZN-PLGA-NPsHG showed a reduced burst release $(30.81$ at 1.5 vs $19.6 \%$ at $1 \mathrm{~h}$, respectively). BZN release from BZN-PLGA-NPs occurred according to two phases. In phase I, the dissolution medium stimulated the release of the drug molecules adsorbed on the surface of the NPs through desorption of particles, in addition, it caused the random break of the polymeric structure causing a reduction in the molecular weight of the polymer. In phase II, a combined erosion/diffusion mechanism took place where the erosion of a thicker polymer layer occurred to allow the diffusion of the drug particles in a slower release rate. For PLGA-NPs-HG, the threedimensional network structure of hydrogels is expected to further delay the release of the drug [47] as the release into the medium is expected to occur in two steps, first the release of the drug from the PLGA-NPs as per the steps mentioned above then the drug followed by drug diffusion through the microchannels of the hydrogel to the external media.

From the analysis of the release profiles (fig. 2), BZN-HG showed faster drug release in comparison to BZN-PLGA-NPs-HGs, in addition, a monophasic zero order release pattern $\left(R^{2}=0.9874\right)$ was reported. In the study conducted by Kassab et al., in 2017, a biphasic release profile was reported for gatifloxacin from periodontal bioadhesive gel. The authors attributed the initial burst effect to the drug which is present freely in the gel matrix [48].

After four hours the HG with free BZN exhibited a drug release of $71.26 \%$, while for BNZ-PLGA-NPs-HG, the maximum amount released was $45.99 \%$. These results evidenced the ability of PLGANPs-HG to offer a sustained drug release of BZN thus, maintaining its presence for a longer time over the affected buccal area. In the study conducted by Ashmoony et al., incorporation of clomipramine in niosomal gel lead to a delayed drug release profile which the authors attributed to the presence of additional diffusion barrier [49].

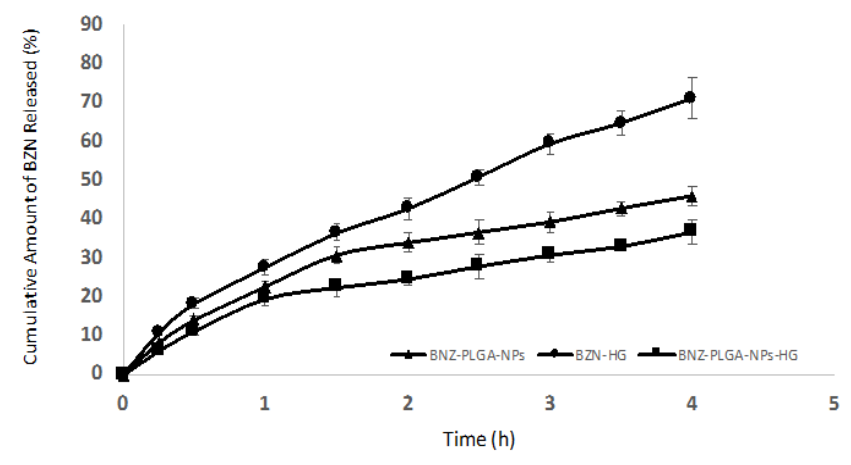

Fig. 2: Drug release profile of BZN from HG, PLGA-NPs and PLGA-NPs-HG (Results are expressed as mean \pm SE, $n=3$ )

\section{In vivo study}

In order to determine the efficacy of tested formulations on the clinical healing of oral mucositis, the ulcer area was measured on consequent observation days and percentage reduction was recorded. The mucositis model used was adapted from Takeuchi $e t$ al., 2018 and Karavanaet al., 2011 [50-51].

In the present study, the most prominent differences between the groups were observed on day 2 . The untreated group showed a worsened ulcer condition. Animal groups 2 and 4 treated with BZN-HG and BZN-PLGA-NPs-HG, respectively showed a significantly higher reduction percentage in ulcer surface area compared to group 3 treated with BZN-PLGA-NPs $\left({ }^{*} p<0.05\right)$, which suggests that both treatments may promote early stage healing [50].

The BZN-PLGA-NPs-HG group 4 needed $10 \mathrm{~d}$ of treatment to complete healing versus $16 \mathrm{~d}, 14 \mathrm{~d}$ and $12 \mathrm{~d}$ for the complete healing of groups 1(no treatment), 3 (treated with BZN-PLGA-NPs) and 2 (BZN-HG).

From these results (table 5), the high retention in the oral cavity of BZNPLGA-NPs-HG could be considered the main factor contributing to its enhanced therapeutic outcome. BZN-PLGA-NPs with non-retentive properties showed the lowest reduction percentage on day 2 and the longest treatment period compared to the other two formulations. BZNHG did not contribute to shortening the treatment period of oral mucositis, however, they helped reduce the ulcer area.

Table 5: Average reduction in ulcer surface area for rabbits in different test groups

\begin{tabular}{|c|c|c|c|c|}
\hline \multirow[t]{2}{*}{ Observation day } & \multicolumn{4}{|c|}{ Reduction in ulcer area (\%) } \\
\hline & $\begin{array}{l}\text { Group } 1^{*} \\
\text { no treatment }\end{array}$ & $\begin{array}{l}\text { Group 2* } \\
\text { BZN-HG }\end{array}$ & $\begin{array}{l}\text { Group } 3^{*} \\
\text { BZN-PLGA-NPs }\end{array}$ & $\begin{array}{l}\text { Group } 4^{*} \\
\text { BZN-PLGA-NPs-HG }\end{array}$ \\
\hline Day 2 & $-34.12 \pm 1.29$ & $39.82 \pm 2.39$ & $17.29 \pm 1.29$ & $41.83 \pm 3.23$ \\
\hline Day 4 & $-5.67 \pm 0.21$ & $45.19 \pm 3.20$ & $39.85 \pm 2.19$ & $60.49 \pm 1.83$ \\
\hline Day 6 & $7.20 \pm 0.88$ & $61.10 \pm 1.93$ & $48.39 \pm 2.71$ & $75.50 \pm 2.01$ \\
\hline Day 8 & $29.10 \pm 2.10$ & $74.84 \pm 5.83$ & $65.30 \pm 4.38$ & $93.02 \pm 4.32$ \\
\hline Day 10 & $48.33 \pm 3.29$ & $89.28 \pm 3.29$ & $83.24 \pm 3.28$ & \\
\hline Day 12 & $70.17 \pm 2.93$ & & $95.29 \pm 6.49$ & \\
\hline Day 14 & $92.10 \pm 4.85$ & & & \\
\hline Day 16 & $100 \pm 5.40$ & & & \\
\hline
\end{tabular}

${ }^{*}$ Results are expressed as mean \pm SD, $n=6$. 


\section{CONCLUSION}

In the present study, PLGA nanoparticles were prepared, drug loaded and incorporated into six different PLX/HPMC HGs. F3 BZNPLGA-NPs-HGs showed promising thermosensitive, mucoadhesive and in vitro release properties and when tested on experimental animals' model, it was able to successfully contribute to the effective treatment of mucositis in a reduced time interval.

\section{AUTHORS CONTRIBUTIONS}

All the authors have contributed equally

\section{CONFLICTS OF INTERESTS}

\section{Declared none}

\section{REFERENCES}

1. Harris DJ. Cancer treatment-induced mucositis pain: strategies for assessment and management. Ther Clin Risk Manag 2006;2:251-8.

2. Berger AM, Fall-Dickson JM. Oral complications. In: Principles and practice of oncology. $7^{\text {th }}$ ed. DeVita VT. Lippincott Williams and Wilkins, Philadelphia, PA, USA; 2005. p. 2523-35.

3. Cinausero M, Aprile G, Ermacora P, Basile D, Vitale MG, Fanotto V, et al. New Frontiers in the pathobiology and treatment of cancer regimen-related mucosal injury. Front Pharmacol 2017;8:354.

4. Al-Ansari S, Zecha JAEM, Barasch A, de Lange J, Rozema FR, Raber Durlacher JE. Oral mucositis induced by conventional cytotoxic cancer therapies is a common and significant clinical problem in oncology. Curr Oral Health Rep 2015;2:202-11.

5. Panahi Y, Ala S, Saeedi M, Okhovatian A, Bazzaz N, Naghizadeh MM. Allopurinol mouth rinse for prophylaxis of fluorouracil-induced mucositis. Eur J Cancer Care 2010;19:308-12.

6. Epsteın JB, Silverman S, Paggiarıno JR, Crockett DA, Schubert S, Senzer MM, et al. Benzy-damine $\mathrm{HCl}$ for prophylaxis of radiation induced oral mucositis: results from a multicenter, randomized, double-blind, placebo-controlled clinical trial. Cancer 2001;92:875-85.

7. De Laat EH, Scholte Op, Reimer WJ, Van Achterberg T. Pressure ulcers: diagnostics and interventions aimed at wound-related complaints: a review of the literature. J Clin Nurs 2005;14:464-72.

8. Danhier F, Ansorena E, Silva JM, Coco R, Breton A, Preat V. PLGA-based nanoparticles: an overview of biomedical applications. J Controlled Release 2012;161:505-22.

9. Abdel Mottaleb MM, Beduneau A, Pellequer Y, Lamprecht A. Stability of fluorescent labels in PLGA polymeric nanoparticles: quantum dots versus organic dyes. Int J Pharm 2015;494:471-8.

10. Zhu X, Zeng X, Zhang X, Cao W, Wang Y, Chen H, et al. The effects of quercetin-loaded PLGA-TPGS nanoparticles on ultraviolet B-induced skin damages in vivo. Nanomed: Nanotechnol Biol Med 2016;12:623-32.

11. Zhou YY, Du YZ, Wang L, Zhou JP, Hu FQ. Preparation and pharmacodynamics of stearic acid and poly (lactic-co-glycolic acid) grafted chitosan oligosaccharide micelles for 10hydroxycamptothecin. Int J Pharm 2010;393:143-51.

12. Rinaldi S, Fortunati E, Taddei M, Kenny JM, Armentano I, Latterini L. Integrated PLGA-agnanocomposite systems to control the degradation rate and antibacterial properties. J Appl Polym Sci 2013;130:1185-93.

13. Bhati R, Nagrajan RK. A detailed review on oral mucosal drug delivery system. Int J Pharm Sci Res 2012;3:659-81.

14. Sohi H, Ahuja A, Ahmad FJ, Khar RK. Critical evaluation of permeation enhancers for oral mucosal drug delivery. Drug Dev Ind Pharm 2010;36:254-82.

15. Hamidi M, Azadi A, Rafiei P. Hydrogel nanoparticles in drug delivery. Adv Drug Delivery Rev 2008;60:1638-49.

16. Shaikh R, Raj Singh TR, Garland MJ, Woolfson AD, Donnelly RF. Mucoadhesive drug delivery systems. J Pharm Bioallied Sci 2011;3:89-100.

17. Priya JH, John R, Alex A, Anoop KR. Smart polymers for the controlled delivery of drugs-a concise overview. Acta Pharm Sin B 2014;4:120-7.

18. Al Khateb K, Ozhmukhametova EK, Mussin MN, Seilkhanov SK, Rakhypbekov TK, Lau WM, et al. In situ gelling systems based on pluronic F127/Pluronic F68 formulations for ocular drug delivery. Int J Pharm 2016;502:70-9.
19. Bujnakova Z, Dutkova E, Balaz M, Turianicova E, Balaz P. Stability studies of As4S4 nanosuspension prepared by wet milling in poloxamer 407. Int J Pharm 2015;478:187-92.

20. De Souza, Ferreir SB, Moco TD, Borghi-Pangoni FB, Junqueira MV, Bruschi ML. Rheological, mucoadhesive and textural properties of thermoresponsive polymer blends for biomedical applications. J Mech Behavior Biomed Mat 2015;55:164-78.

21. Dewan M, Bhowmick B, Sarkar G, Rana D, Bain MK, Bhowmik $\mathrm{M}$, et al. Effect of methyl cellulose on gelation behavior and drug release from poloxamer based ophthalmic formulations. Int J Bio Macromol 2015;72:706-10.

22. Loh XJ, Goh SH, Li J. New biodegradable thermo-gelling copolymers having very low gelation concentrations. Biomacromology 2007;8:585-93.

23. Koffi AA, Agnely F, Ponchelb G, Grossiordb JL. Modulation of the rheological and mucoadhesive properties of thermosensitive poloxamer-based hydrogels intended for the rectal administration of quinine. Eur J Pharm Sci 2006;27:328-35.

24. Anlar S, Capan Y, Guven O, Gogus A, Dalkara T, Hincal AA. Formulation and in vitro-in vivo evaluation of buccoadhesive morphine sulphate tablets. Pharm Res 1994;11:231-6.

25. Mashru RC, Sutariya VB, Sankalia MG, Parikh PP. Development and evaluation of fast-dissolving film of salbutamol sulphate. Drug Dev Ind Pharm 2005;31:25-34.

26. Gohel MC, Parikh RK, Aghara PY, Nagori SA, Delvadia RR, Dabhi M. Application of simplex lattice design and desirability function for the formulation development of mouth dissolving film of salbutamol sulphate. Curr Drug Delivery 2009;6:486-94.

27. de Sa FA, Taveira SF, Gelfuso GM, Lima EM, Gratieri T. Liposomal voriconazole (VOR) formulation for improved ocular delivery. Colloids Surf B 2015;133:331-8.

28. Shivakumar $\mathrm{H}$, Yadav $\mathrm{H}$. In vitro and in vivo evaluation of $\mathrm{pH}-$ sensitive hydrogels of carboxymethyl chitosan for intestinal delivery of theophylline. ISRN Pharm 2012;7:127-45.

29. Shimamura Y, Takeuchi I, Terada H, Makino K. A mouse model for oral mucositis induced by cancer chemotherapy. Anticancer Res 2018;38:307-12.

30. Choipang C, Chuysinuan P, Suwantong O, Ekabutr P, Supaphol P. Hydrogel wound dressings loaded with PLGA/ciprofloxacin hydrochloride nanoparticles for use on pressure ulcers. J Drug Delivery Sci Tech 2018;47:106-14.

31. Jeong YI, Na HS, Seo DH, Kim DG, Lee HC, Jang MK, et al. Ciprofloxacin-encapsulated poly (DL-lactide-co-glycolide) nanoparticles and its antibacterial activity. Int J Pharm 2008;352:31723.

32. Sun L, Liu Z, Wang L, Cun D, Tong HHY, Yan R, et al. Enhanced topical penetration, system exposure and anti-psoriasis activity of two particle-sized, curcumin-loaded PLGA nanoparticles in hydrogel. J Controlled Release 2017;254:45-54.

33. Abrego G, Alvarado HL, Egea MA, Gonzalez-Mira E, Calpena AC, Garcia ML. Design of nanosuspensions and freeze-dried PLGA nanoparticles as a novel approach for ophthalmic delivery of pranoprofen. J Pharm Sci 2014b;103:3153-64.

34. Tugcu Demi roz F. Development of in situ poloxamer-chitosan hydrogels for vaginal drug delivery of benzydamine hydrochloride: Textural, mucoadhesive and in vitro release properties. Marmara Pharm J 2017;21:762-70.

35. Joshi M, Bolmal U, Dandagi P. Formulation and evaluation of cefuroxime axetil sol gel for periodontits. Int J Pharm Pharm Sci 2014;6:498-503.

36. Liu T, Chu B. Formation of homogeneous gel-like phases by mixed triblock copolymer micelles in aqueous solution: FCC to BCC phase transition. J Appl Cryst 2000;33:727-30.

37. Cabana A, Ait-Kadi A, Juhasz J. Study of gelation process of polyethylene oxidea. J Colloid Interface Sci 1997;190:307-12.

38. Russo E, Selmin F, Baldassari S, Gennari CGM, Caviglioli G, Cilurzo F, et al. A focus on mucoadhesive polymers and their application in buccal dosage forms. J Drug Delivery Sci Technol 2016;32:113-25.

39. Smart JD, Kellaway IW, Worthington HE. An in vitro investigation of mucosa-adhesive materials for use in controlled drug delivery. J Pharm Pharmacol 1984;36:295-9.

40. Gu JM, Robinson JR, Leung SH. Binding of acrylic polymers to mucin/epithelial surfaces: Structure-property relationships. Crit Rev Ther Drug Carrier Syst 1988;5:21-67. 
41. Ponchel G, Touchard F, Duchene D, Peppas NA. Bioadhesive analysis of controlled-release systems. I Fracture and interpenetration analysis in poly (acrylic acid)-containing systems. J Controlled Release 1987;5:129-41.

42. Parhi R. Development and optimization of pluronic ${ }^{\circledR}$ F127 and HPMC based thermosensitive gel for the skin delivery of metoprolol succinate. J Drug Delivery Sci Tech 2016;36:23-33.

43. Bader RA, Putnam DA. Engineering polymer systems for improved drug delivery. New Jersy, USA: Wiley; 2013.

44. Chatterjee B, Amalina N, Sengupta P, Mandal UK. Mucoadhesive polymers and their mode of action: a recent update. J Appl Pharm Sci 2017; 7:195-203.

45. dos Santos TC, Rescignano N, Boff L, Reginatto FH, Simoes CMO, de Campos AM, et al. Manufacture and characterization of chitosan/PLGA nanoparticles nanocomposite buccal films. Carbohydrate Polymers 2017;173:638-44.

46. Dalpiaz A, Sacchetti F, Baldisserotto A, Pavan B, Maretti E, Iannuccelli V, et al. Application of the "in-oil nanoprecipitation" method in the encapsulation of hydrophilic drugs in PLGA nanoparticles. J Drug Delivery Sci Tech 2016;32:283-90.

47. Bhattarai N, Gunn J, Zhang M. Chitosan-based hydrogels for controlled, localized drug delivery. Adv Drug Delivery Rev 2010;62:83-99.

48. Kassab HJ, Thomas LM, Jabir SA. Development and physical characterization of a periodontal bioadhesive gel of gatifloxacin. Int J Appl Pharm 2017;9:31-6.

49. Mohawed OAM, Ashmoony MM, Gazayerly ON. Niosomeencapsulated clomipramine for transdermal controlled delivery. Int J Pharm Pharm Sci 2014;6:567-75.

50. Takeuchi I, Kamiki Y, Makino K. Therapeutic efficacy of rebamipide-loaded PLGA nanoparticles coated with chitosan in mouse model for oral mucositis induced by cancer chemotherapy. Colloids Surf B 2018;167:468-73.

51. Karavana (Hizarcioglu) SY, Sezer B, Guneri P, Veral A, Boyacioglu H, Ertan G, et al. Efficacy of topical benzydamine hydrochloride gel on oral mucosal ulcers: an in vivo animal study. Int J Oral Maxillofac Surg 2011;40:973-8. 